\title{
Mudanças na composição e adequação nutricional da dieta familiar nas áreas me- tropolitanas do Brasil (1988-1996)
}

Secular changes in dietary patterns in the metropolitan areas of Brazil (1988-1996)

Carlos Augusto Monteiro ${ }^{\mathrm{a}, \mathrm{c}}$, Lenise Mondini ${ }^{\mathrm{b}}$ e Renata BL Costa

${ }^{a}$ Departamento de Nutrição da Faculdade de Saúde Pública da Universidade de São Paulo. São Paulo, SP, Brasil. ' Departamento de Medicina Preventiva da Universidade Federal de São Paulo. São Paulo, SP, Brasil. ' Núcleo de Pesquisas Epidemiológicas em Nutrição e Saúde da Universidade de São Paulo. São Paulo, SP, Brasil 


\title{
Mudanças na composição e adequação nutricional da dieta familiar nas áreas metropolitanas do Brasil (1988-1996) Secular changes in dietary patterns in the metropolitan areas of Brazil (1988-1996)
}

\author{
Carlos Augusto Monteiro ${ }^{\mathrm{a}, \mathrm{c}}$, Lenise Mondini ${ }^{\mathrm{b}}$ e Renata BL Costa \\ aDepartamento de Nutrição da Faculdade de Saúde Pública da U niversidade de São Paulo. São \\ Paulo, SP, Brasil. ' Departamento de Medicina Preventiva da Universidade Federal de São Paulo. São \\ Paulo, SP, Brasil. cNúcleo de Pesquisas Epidemiológicas em N utrição e Saúde da U niversidade de São \\ Paulo. São Paulo, SP, Brasil
}

\section{Descritores}

Inquéritos sobre dietas". Hábitos alimentares". Consumo de alimentos". Necessidades nutricionais. Dieta, economia. Zonas metropolitanas. Tendência secular.

\section{Resumo}

\section{Objetivo}

Atualizar a tendência secular (1962-1988) da composição e da adequação nutricional da dieta familiar praticada nas áreas metropolitanas do Brasil, com base em nova pesquisa sobre orçamentos familiares realizada em meados da década de 90 .

\section{Métodos}

Utilizaram-se como fontes de dados as pesquisas sobre orçamentos familiares (POF) da Fundação IBGE, realizadas entre março de 1987 e fevereiro de 1988 (13.611 domicílios) e entre outubro de 1995 e setembro de 1996 (16.014 domicílios), tendo ambas como universo de estudo as áreas metropolitanas do Brasil. Nas duas pesquisas chegou-se à disponibilidade domiciliar diária per capita de alimentos, dividindo-se o total de alimentos adquiridos no mês pelo número de pessoas residentes no domicílio e pelo número de dias do mês. O padrão alimentar foi caracterizado com base na participação relativa de grupos selecionados de alimentos e de nutrientes na disponibilidade calórica total. A comparação entre as duas pesquisas levou em conta o conjunto das áreas metropolitanas do País e estratos dessas áreas correspondentes às regiões menos desenvolvidas (Norte e Nordeste) e mais desenvolvidas (Centro-Oeste, Sudeste e Sul).

\section{Resultados}

Observou-se intensificação do consumo relativo de carnes, de leites e de seus derivados (exceto manteiga) em todas as áreas metropolitanas, enquanto o consumo de ovos passou a declinar, sobretudo no Centro-Sul do País. Leguminosas, raízes e tubérculos prosseguiram sua trajetória descendente, mas cereais e derivados tenderam a se estabilizar no Centro-Sul ou mesmo a se elevar ligeiramente no NorteNordeste. A participação relativa de açúcar refinado e refrigerantes cresceu em todas as áreas, sendo que a participação de óleos e gorduras vegetais manteve-se constante no Norte-Nordeste e declinou intensamente no Centro-Sul.

\section{Conclusões}

A tendência ascendente da participação relativa de lipídios na dieta do Norte e do Nordeste, o aumento no consumo de ácidos graxos saturados em todas as áreas metropolitanas do País, ao lado da redução do consumo de carboidratos completos, da estagnação ou da redução do consumo de leguminosas, verduras, legumes e frutas e do aumento no consumo já excessivo de açúcar são os traços marcantes e negativos da evolução do padrão alimentar entre 1988 e 1996. Mudanças que podem indicar a adesão da população a dietas mais saudáveis - declínio no consumo de ovos e recuo discreto da elevada proporção de calorias lipídicas - foram registradas apenas no Centro-Sul do País.
Correspondência para/Correspondence to:

Carlos Augusto Monteiro

Av. Dr. Arnaldo, 715

01246-904, São Paulo, SP, Brasil

E-mail:carlosam@usp.br
Edição subvencionada pela Fapesp (Processo n ${ }^{\circ} 00 / 01601-8$ ).

Recebido em 3/1/2000. Reapresentado em 28/4/2000. Aprovado em 4/5/2000. 


\section{Keywords}

Diet surveys. Food habits . Food $^{\#}$ consumptions $^{\#}$. Nutritional requirements. Diet, economy. Metropolitan zones, Brazil. Secular trends.

\begin{abstract}
Objective

A new family budget survey carried out in the mid-nineties in Brazil allows an update of the secular trends (1962-1988) of dietary patterns of Brazilian population living in metropolitan areas.

Methods

Data sources are IBGE Institute of Statistics family budget surveys carried out from March 1987 to February 1988(13,611 households) and from October 1995 to September 1996 (16,014 households) in all metropolitan areas of Brazil. The daily food availability per capita for each household was calculated dividing the total food acquired in a month by the number of individuals living in a household and the month's number of days. Dietary patterns were characterized according to the amount of selected food groups and nutrients relative to the diet caloric input. Comparisons between the two surveys included the metropolitan area population as a whole and subgroups from less (North and Northeast) and more developed (Mid-west, Southeast and South) regions.

Results

It was observed an increase in consumption of meat and dairy products (except for butter) and a reduction in eggs consumption in both less and more developed regions. Beans, roots and tubers consumption showed a steady decline in the whole country while cereals consumption remained the same (higher in developed regions) or had a slight increase (in less developed regions). The proportional consumption of vegetal oils and margarine remained constant in the less developed regions but their consumption was greatly reduced in the more developed ones.

Conclusions

An increase in the diet's lipid content in less developed regions and of saturated fat in the country as a whole, associated with a decrease or even no consumption of beans, vegetables, fruits and complex carbohydrates, and a further increase in the excessive sugar consumption are the negative aspects of the trend observed from 1988 to 1996. Changes that may indicate a growing awareness of the population toward a healthier diet, such as a decline in egg intake and a slight reduction in diets with a high total lipid content, were found only in more developed regions.
\end{abstract}

\section{INTRODUÇÃO}

Os danos para a saúde que podem decorrer do consumo insuficiente de alimentos - desnutrição - ou do consumo excessivo - obesidade - são há muito conhecidos pelos seres humanos. Apenas mais recentemente, entretanto, acumulam-se evidências de que características qualitativas da dieta são igualmente importantes na definição do estado de saúde, em particular no que se refere a doenças crônicas da idade adulta. A relação entre consumo de gorduras saturadas, níveis plasmáticos de colesterol e risco de doença coronariana foi das primeiras a ser comprovada empiricamente. ${ }^{13,14}$ Assim como ocorre com as gorduras saturadas, o consumo elevado de colesterol também pode aumentar o risco de doença coronariana. ${ }^{20} \mathrm{O}$ risco do câncer de mama, próstata e cólon-reto parece aumentar com o consumo total de gordura e com o consumo de gordura de origem animal, enquanto dietas com legumes, verduras e frutas cítricas, ou seja, ricas em fibras, vitaminas e minerais, certamente protegem os indivíduos de cânceres de pulmão, boca, faringe, esôfago, estômago e cólonreto. ${ }^{23}$ Inúmeras evidências bioquímicas, microbiológicas, clínicas e epidemiológicas apontam uma relação causal indiscutível entre consumo freqüente de açúcar e cárie dental. ${ }^{21}$ Em nível populacional, demonstra-se uma forte associação entre o nível do consumo habitual de sal e a pressão arterial dos indivíduos. ${ }^{11}$ Embora o consumo leve para moderado de certos tipos de bebidas alcoólicas pareça conferir alguma proteção contra a doença coronariana, o consumo moderado ou intenso está associado a aumento no risco de grande número de enfermidades, incluindo a hipertensão arterial e a doença cérebro-vascular, doenças do fígado, certos tipos de câncer, osteoporose, defeitos congênitos (no caso de consumo intenso durante a gravidez) e de mortes por acidentes e violências. ${ }^{21,23}$ Dietas ricas em carboidratos complexos são consideradas úteis na prevenção da obesidade e de vários tipos de câncer e no controle da hiperlipidemia e do diabetes mellitus. ${ }^{21}$ A maior parte dos estudos sobre a relação entre características da dieta e obesidade evidencia uma associação positiva entre proporção da energia procedente de gorduras e risco de obesidade, ainda que as associações obtidas em estudos populacionais tendam a alcançar magnitude inferior à evidenciada por estudos experimentais. ${ }^{2} \mathrm{~A}$ obesidade, por sua vez, aumenta o risco de diversas doen- 
ças crônicas da idade adulta, destacando-se as doenças cardiovasculares, o diabetes mellitus tipo II, as disfunções biliares, os problemas do aparelho locomotor e certos tipos de câncer. ${ }^{22} \mathrm{O}$ acúmulo de evidências que associam características da dieta ao estado de saúde dos indivíduos determinou que a Organização Mundial de Saúde estabelecesse limites populacionais máximos para o consumo de gorduras (30\% do consumo calórico total), ácidos graxos saturados (10\% do consumo calórico total), açúcar (10\% do consumo calórico total), colesterol (300 mg por dia ou $100 \mathrm{mg} / 1.000 \mathrm{kcal})$ e sal ( $6 \mathrm{~g}$ por dia), e que estimulasse o consumo de carboidratos complexos (mínimo de $50 \%$ do consumo calórico total) e de legumes, verduras e frutas (400 g por dia ou cerca de $7 \%$ do consumo calórico total). ${ }^{12,21}$

A investigação direta do consumo alimentar a partir da aplicação de inquéritos dietéticos constitui a forma ideal para se caracterizar os padrões dietéticos vigentes em uma dada população e sua evolução ao longo do tempo. Entretanto, a grande variabilidade que usualmente caracteriza o consumo alimentar dos indivíduos exige o estudo de grandes amostras por períodos relativamente longos de tempo, condição que encarece os inquéritos dietéticos e os torna pouco factíveis. Uma das alternativas utilizadas com frequiência para se estimar a situação e a evolução de padrões dietéticos são os dados nacionais sobre disponibilidade de alimentos. Esses dados, compilados anualmente pela Organização das Nações Unidas para a Alimentação e a Agricultura (FAO), indicam a quantidade potencial média de alimentos disponíveis para consumo humano em cada país e são calculados com base em estimativas da produção, da importação e da exportação de produtos alimentares e em um percentual arbitrado para o desperdício. Séries históricas da disponibilidade de alimentos não permitem qualquer desagregação das estimativas nacionais, além de dependerem grandemente da qualidade das estatísticas nacionais referentes à produção e à comercialização dos alimentos. Uma melhor alternativa, na ausência de inquéritos dietéticos, é representada pelas pesquisas de orçamentos familiares (POF). As POF são inquéritos domiciliares que, a partir do levantamento sistemático dos gastos com alimentos e dos preços praticados nos locais de compra desses alimentos, permitem estimar a disponibilidade individual de alimentos de cada família. São limitações das POF, entretanto, a não-consideração da fração desperdiçada dos alimentos, o não-registro dos alimentos doados ou consumidos fora do domicílio e a inexistência de informações sobre a distribuição intrafamiliar dos alimentos. ${ }^{10}$

Em trabalho prévio, baseado em pesquisas metropolitanas de orçamento familiar realizadas nas dé- cadas de $60^{9}$ e $80^{5}$ e em um inquérito nacional sobre consumo alimentar realizado na década de $70,{ }^{4}$ foram reunidos elementos que permitiram caracterizar a evolução de padrões de alimentação da população urbana do Brasil em um período de quase trinta anos. ${ }^{16}$ Importantes mudanças com reflexos evidentes para o perfil nutricional da população puderam ser detectadas, incluindo-se o aumento no consumo de produtos de origem animal (principalmente leite e derivados, mas também carnes e ovos) em detrimento de cereais, feijão, raízes e tubérculos, e a intensa substituição de gorduras animais (banha, toucinho e manteiga) por óleos vegetais e margarinas. Uma nova pesquisa metropolitana de orçamentos familiares, realizada pela Fundação IBGE em 1995-1996, possibilita a atualização das tendências temporais do consumo alimentar nas áreas metropolitanas do País, sendo este o objetivo principal do presente trabalho.

\section{MÉTODOS}

As fontes de dados utilizadas no presente estudo são as pesquisas metropolitanas nacionais sobre orçamentos familiares da Fundação IBGE, realizadas entre março de 1987 a fevereiro de 1988 (POF-1988) e entre outubro de 1995 e setembro de 1996 (POF-1996). ${ }^{8}$ O universo de estudo das duas pesquisas foi o mesmo, compreendendo as áreas metropolitanas de Belém, Fortaleza, Recife, Salvador, Belo Horizonte, Rio de Janeiro, São Paulo, Curitiba, Porto Alegre, Brasília e o Município de Goiânia. Na POF-1988 foram estudados 13.611 domicílios e na POF-1996, 16.014 domicílios. ${ }^{5,7}$

Na POF-1988, a quantidade de alimentos disponível para consumo no domicílio foi estimada com base na divisão do gasto mensal declarado pelas famílias com cada tipo de alimento pelo preço médio de varejo do alimento no momento do estudo. Na POF-1996, a quantidade de alimento disponível para consumo foi estimada diretamente a partir das quantidades declaradas de alimentos compradas pelas famílias. Nas duas pesquisas chegou-se à disponibilidade domiciliar diária per capita de alimentos dividindose o total de alimentos adquiridos no mês pelo número de pessoas residentes no domicílio e pelo número de dias do mês. ${ }^{5,7}$

O padrão alimentar da população, nos períodos de realização das duas pesquisas, foi caracterizado com base na participação relativa de grupos de alimentos e de nutrientes selecionados na disponibilidade diária per capita de energia. Quinze grupos de alimentos foram considerados: cereais e derivados; leguminosas e derivados; verduras e legumes; raízes, tubérculos e derivados; carnes e embutidos; leite e derivados; açú- 
car e refrigerantes; óleos e gorduras vegetais; frutas e sucos naturais; oleaginosas; ovos; banha, toucinho e manteiga; bebidas alcoólicas; condimentos; outros alimentos e preparações. A participação relativa de nutrientes na dieta levou em conta a proporção de energia decorrente de açúcar refinado (sacarose) e de outros carboidratos, de proteínas de origem animal e vegetal, de lipídios de origem animal e vegetal, de ácidos graxos saturados, monoinsaturados e poliinsaturados, e de colesterol.

A comparação do padrão alimentar identificado nas duas pesquisas - participação relativa de alimentos e nutrientes no total calórico disponível - levou em conta o conjunto das áreas metropolitanas do País e dois subconjuntos que correspondem às áreas metropolitanas das regiões menos desenvolvidas (Norte e Nordeste) e mais desenvolvidas (Sudeste, Sul e Centro-Oeste) do País. No primeiro grupo (Norte-Nordeste) encontram-se Belém, Fortaleza, Recife e Salvador; no segundo estão Belo Horizonte, Rio de Janeiro, São Paulo, Curitiba, Porto Alegre, Brasília e o Município de Goiânia (Centro-Sul). De acordo com a POF-1996, famílias com renda inferior a dois salários-mínimos representavam $18,6 \%$ do total de famílias existentes nas áreas metropolitanas do Norte-Nordeste e apenas 5,8\% do total de famílias metropolitanas do Centro-Sul. Por outro lado, rendas familiares acima de trinta salários-mínimos eram encontradas em 6,6\% das famílias metropolitanas do Norte-Nordeste e em 12,8\% das famílias metropolitanas do Centro-Sul. ${ }^{7}$

A disponibilidade diária per capita de energia apurada pelas pesquisas de orçamento familiar realizadas em 1988 e 1996 foi de, respectivamente, 1.919,0 kcal e 1.711,2 kcal. Os mesmos valores foram de $1.704,4 \mathrm{kcal}$ e $1.706,1 \mathrm{kcal}$ para as áreas metropolita- nas das Regiões Norte e Nordeste e de $1.965,1$ kcal e $1.712,4 \mathrm{kcal}$ para as áreas metropolitanas das regiões do Centro-Sul.

A transformação de quantidades de alimentos em nutrientes foi feita por um aplicativo desenvolvido no Departamento de Nutrição da Faculdade de Saúde Pública da Universidade de São Paulo, que utiliza a tabela de composição ENDEF ${ }^{6}$ suplementada, no caso específico da análise de frações lipídicas, por tabelas americanas de composição alimentar. ${ }^{1,15,19}$

\section{RESULTADOS}

A Tabela 1 compara a importância relativa de quinze grupos de alimentos nas pesquisas de 1988 e 1996. Cada grupo de alimentos aparece em cada pesquisa com a proporção que lhe corresponde na disponibilidade total de energia conferida pelo conjunto dos alimentos.

Nas áreas metropolitanas das Regiões Norte e Nordeste, o que mais chama a atenção é o aumento na importância relativa das carnes (de 12,5\% para 14,1\% do total calórico) e a expansão do grupo de cereais e derivados (de 30,6\% para 32,9\%) em detrimento do grupo de raízes e tubérculos (de 12,1\% para 8,9\%). Embora de menores dimensões, merecem registro o declínio no consumo relativo de frutas e de sucos naturais (de 3,3\% para 2,4\%) e o aumento na contribuição calórica proveniente do açúcar refinado e dos refrigerantes (de 13,5\% para 13,9\%). Nas duas pesquisas, a contribuição calórica do açúcar refinado excede o limite máximo recomendado de $10 \%$, enquanto o consumo relativo de legumes, verduras e frutas fica bem abaixo do limite mínimo de $7 \%$.

Nas áreas metropolitanas das regiões do CentroSul, as alterações que mais se destacam são o au-

Tabela 1 - Participação relativa (\%) de grupos de alimentos na disponibilidade total de energia. Áreas metropolitanas do Brasil, 1988 e 1996.

\begin{tabular}{|c|c|c|c|c|c|c|}
\hline \multirow[b]{2}{*}{ Grupos de alimentos } & \multicolumn{2}{|c|}{ Norte-Nordeste } & \multicolumn{2}{|c|}{ Centro-Sul } & \multicolumn{2}{|c|}{ Brasil } \\
\hline & 1988 & 1996 & 1988 & 1996 & 1988 & 1996 \\
\hline Cereais e derivados & 30,6 & 32,9 & 35,0 & 35,3 & 34,4 & 34,8 \\
\hline Leguminosas e derivados & 7,4 & 7,3 & 5,6 & 5,3 & 5,8 & 5,7 \\
\hline Verduras e legumes & 0,5 & 0,5 & 0,6 & 0,5 & 0,6 & 0,5 \\
\hline Raízes, tubérculos e derivados & 12,1 & 8,9 & 3,2 & 2,7 & 4,6 & 4,0 \\
\hline Carnes e embutidos & 12,5 & 14,1 & 10,5 & 13,0 & 10,8 & 13,2 \\
\hline Leites e derivados & 5,7 & 6,0 & 8,4 & 8,9 & 8,0 & 8,2 \\
\hline Açúcar e refrigerantes & 13,5 & 13,9 & 13,2 & 13,5 & 13,2 & 13,7 \\
\hline Óleos e gorduras vegetais & 10,0 & 10,0 & 15,2 & 12,9 & 14,4 & 12,4 \\
\hline Frutas e sucos naturais & 3,3 & 2,4 & 3,2 & 3,2 & 3,2 & 3,0 \\
\hline Oleaginosas & 0,3 & 0,2 & 0,1 & 0,1 & 0,2 & 0,1 \\
\hline Ovos & 1,5 & 1,3 & 1,5 & 1,0 & 1,5 & 1,0 \\
\hline Banha, toucinho e manteiga & 0,7 & 0,6 & 0,9 & 0,7 & 0,9 & 0,7 \\
\hline Bebidas alcoólicas & 0,4 & 0,5 & 0,5 & 0,6 & 0,5 & 0,6 \\
\hline Condimentos & 0,2 & 0,3 & 0,4 & 0,4 & 0,4 & 0,4 \\
\hline Outras preparações & 1,2 & 1,0 & 1,7 & 1,8 & 1,6 & 1,6 \\
\hline Total & 100,0 & 100,0 & 100,0 & 100,0 & 100,0 & 100,0 \\
\hline
\end{tabular}

Fonte: Fundação IBGE (1999). 
mento na importância relativa das carnes (de 10,5\% para $13 \%$ do total calórico) e a redução da participação de óleos e de gorduras vegetais (de 15,2\% para $12,9 \%)$ e, em menor grau, de raízes e tubérculos (de 3,2\% para 2,7\%). Digno de nota, sobretudo por apontar direção oposta à observada com relação às carnes (e também a leite e derivados), é a queda pronunciada no consumo de ovos $(1,5 \%$ do total calórico em 1988 e $1 \%$ em 1996). Nota-se, ainda, pequeno declínio na disponibilidade relativa de feijão e das demais leguminosas, virtual estagnação quanto a cereais, verduras, legumes, frutas e sucos naturais, e ligeira ascensão na proporção de calorias provenientes do açúcar e dos refrigerantes. Também no Centro-Sul, nas duas pesquisas, é excessivo o consumo de açúcar e insuficiente o consumo de legumes, verduras e frutas.

Levando-se em conta o conjunto das áreas metropolitanas do País, as mudanças de maior magnitude são o aumento relativo na oferta de carnes (de 10,8\% para 13,2\%) e a redução na participação de óleos e gorduras vegetais (de $14,4 \%$ para $12,4 \%$ ). Na Tabela 2 detalha-se a evolução individual dos alimentos que integram os quinze grupos de alimentos focalizados no presente estudo.*

A Tabela 3 descreve modificações na composição da disponibilidade alimentar com relação a macronutrientes. Nota-se que a participação de carboidratos nas dietas tende a declinar entre as pesquisas nas áreas metropolitanas do Norte-Nordeste, sendo esse declínio compensado, em partes semelhantes, pelo aumento na oferta de proteínas e de lipídios. No Centro-Sul chamam a atenção a manutenção da participação de carboidratos na dieta e a substituição de lipídios por proteínas. Embora ascendente, a participação de lipídios no Norte e Nordeste (23\% do total calórico em 1987 e 23,8\% em 1996) ainda é bem inferior à observada no CentroSul (29,5\% em 1988 e 28,4\% em 1996). A participação dos lipídios na dieta do Centro-Sul ultrapassaria $30 \%$, limite máximo preconizado para o consumo de gorduras, ${ }^{21}$ não fosse o declínio intenso do consumo de óleos e de gorduras vegetais registrado entre as duas pesquisas.

A Tabela 4 fornece detalhes adicionais sobre mudanças observadas nas áreas metropolitanas do País quanto à oferta alimentar de carboidratos, de proteínas e de lipídios. Em todo o País cresce a proporção das proteínas que procedem de fontes animais (cerca de 60\% em 1988 e de 63\% em 1996). Os lipídios de origem animal também aumentam nas duas regiões, embora um aumento expressivo ocorra apenas nas
Tabela 2 - Participação relativa (\%) de alimentos e grupos de alimentos na disponibilidade de energia. Áreas metropolitanas do Brasil, 1988 e 1996.

\begin{tabular}{|c|c|c|}
\hline \multirow[b]{2}{*}{ Grupos de alimentos } & \multicolumn{2}{|c|}{ Brasil Metropolitano } \\
\hline & 1988 & 1996 \\
\hline Cereiais e derivados: & 34,4 & 34,8 \\
\hline · Arroz polido & 15,8 & 15,7 \\
\hline · Pão francês & 7,9 & 8,2 \\
\hline - Biscoitos & 1,9 & 2,7 \\
\hline - Macarrão & 2,5 & 2,7 \\
\hline - Farinha de trigo & 2,1 & 1,9 \\
\hline - Outros & 4,2 & 3,6 \\
\hline Leguminosas e derivados: & 5,8 & 5,7 \\
\hline · Feijão & 5,8 & 5,7 \\
\hline - Outros & 0,0 & 0,0 \\
\hline Verduras e legumes: & 0,6 & 0,5 \\
\hline . Tomate & 0,2 & 0,2 \\
\hline Outros & 0,4 & 0,3 \\
\hline Raízes, tubérculos e derivados: & 4,6 & 4,0 \\
\hline - Batata inglesa & 1,1 & 0,9 \\
\hline - Mandioca e derivados & 2,7 & 2,5 \\
\hline - Outros & 0,8 & 0,6 \\
\hline Carnes: & 10,8 & 13,2 \\
\hline - Bovina & 5,3 & 6,4 \\
\hline - Frango & 2,5 & 3,2 \\
\hline · Suína & 1,4 & 0,9 \\
\hline - Peixes & 0,6 & 0,6 \\
\hline - Embutidos & 0,7 & 1,7 \\
\hline - Outras & 0,3 & 0,4 \\
\hline Leites e derivados: & 8,0 & 8,2 \\
\hline - Leite pasteurizado & 5,4 & 5,0 \\
\hline - Leite em pó integral & 0,6 & 1,1 \\
\hline - Queijos & 1,1 & 1,4 \\
\hline - Outros & 0,9 & 0,7 \\
\hline Açúcar e refrigerantes: & 13,2 & 13,7 \\
\hline - Açúcar & 12,4 & 12,4 \\
\hline - Refrigerantes & 0,8 & 1,3 \\
\hline Óleos e gorduras vegetais: & 14,4 & 12,4 \\
\hline · Óleo de soja & 11,3 & 10,1 \\
\hline - Margarina & 2,5 & 1,8 \\
\hline - Outros & 0,6 & 0,5 \\
\hline Frutas e sucos naturais: & 3,2 & 3,0 \\
\hline - Bananas & 1,1 & 0,9 \\
\hline - Laranjas & 1,1 & 1,0 \\
\hline - Outras & 1,0 & 1,1 \\
\hline Oleaginosas: & 0,2 & 0,1 \\
\hline$\cdot \mathrm{Coco}$ & 0,1 & 0,1 \\
\hline - Outras & 0,1 & 0,0 \\
\hline Ovos & 1,5 & 1,0 \\
\hline Gordura animal: & 0,9 & 0,7 \\
\hline - Manteiga & 0,5 & 0,4 \\
\hline - Toucinho & 0,4 & 0,3 \\
\hline Bebidas alcoólicas: & 0,5 & 0,6 \\
\hline - Cerveja & 0,3 & 0,4 \\
\hline - Aguardente & 0,1 & 0,1 \\
\hline - Outras & 0,1 & 0,1 \\
\hline Condimentos & 0,4 & 0,4 \\
\hline Outras preparações & 1,6 & 1,6 \\
\hline Total & 100,0 & 100,0 \\
\hline
\end{tabular}

áreas metropolitanas do Centro-Sul (de 39\% para $44,4 \%$ ), onde, como se viu, aumentos importantes na oferta de carnes, de leite e seus derivados ocorreram em paralelo a reduções também importantes no consumo de óleos e de gorduras vegetais. A fração do açúcar refinado (sacarose) cresce sobre a fração dos demais carboidratos, tanto no Norte-Nordeste quanto no Centro-Sul. Nas duas regiões, a proporção das calorias totais provenientes de carboidratos complexos, os quais representam a imensa maioria dos "demais carboidratos", fica bastante aquém do limite mínimo recomendado de $50 \%$. 
Tabela 3 - Participação relativa (\%) de macronutrientes na disponibilidade total de energia. Áreas metropolitanas do Brasil, 1988 e 1996.

\begin{tabular}{lcccccc}
\hline & \multicolumn{2}{c}{ Norte-Nordeste } & \multicolumn{2}{c}{ Centro-Sul } & \multicolumn{2}{c}{ Brasil } \\
\cline { 2 - 7 } Macronutriente & 1988 & 1996 & 1988 & 1996 & 1988 & 1996 \\
\hline Carboidratos & 62,6 & 60,7 & 57,2 & 57,0 & 58,1 & 57,8 \\
Proteínas & 14,4 & 15,5 & 13,3 & 14,6 & 13,5 & 14,7 \\
Lipídios & 23,0 & 23,8 & 29,5 & 28,4 & 28,4 & 27,5 \\
\hline Total & 100,0 & 100,0 & 100,0 & 100,0 & 100,0 & 100,0 \\
\hline
\end{tabular}

Fonte: Fundação IBGE $^{8}$ (1999).

Tabela 4 - Disponibilidade de macronutrientes segundo a origem. Áreas metropolitanas do Brasil, 1988 e 1996.

\begin{tabular}{|c|c|c|c|c|c|c|}
\hline \multirow[b]{2}{*}{ Macronutriente } & \multicolumn{2}{|c|}{ Norte-Nordeste } & \multicolumn{2}{|c|}{ Centro-Sul } & \multicolumn{2}{|c|}{ Brasil } \\
\hline & 1988 & 1996 & 1988 & 1996 & 1988 & 1996 \\
\hline $\begin{array}{l}\text { Carboidratos: } \\
. \% \text { Açúcar (Sacarose) } \\
. \% \text { Demais carboidratos }\end{array}$ & $\begin{array}{l}21,9 \\
78,1\end{array}$ & $\begin{array}{l}23,4 \\
76,6\end{array}$ & $\begin{array}{l}23,4 \\
76,6\end{array}$ & $\begin{array}{l}24,1 \\
75,9\end{array}$ & $\begin{array}{l}23,2 \\
76,8\end{array}$ & $\begin{array}{l}24,2 \\
75,8\end{array}$ \\
\hline $\begin{array}{l}\text { Proteínas: } \\
\quad . \% \text { Animal } \\
\quad . \% \text { Vegetal }\end{array}$ & $\begin{array}{l}60,1 \\
39,9\end{array}$ & $\begin{array}{l}62,6 \\
37,4\end{array}$ & $\begin{array}{l}59,2 \\
40,8\end{array}$ & $\begin{array}{l}63,1 \\
36,9\end{array}$ & $\begin{array}{l}59,4 \\
40,6\end{array}$ & $\begin{array}{l}63,1 \\
36,9\end{array}$ \\
\hline $\begin{array}{l}\text { Lipídios: } \\
. \% \text { Animal } \\
. \% \text { Vegetal }\end{array}$ & $\begin{array}{l}43,4 \\
56,6\end{array}$ & $\begin{array}{l}44,9 \\
55,1\end{array}$ & $\begin{array}{l}39,0 \\
61,0\end{array}$ & $\begin{array}{l}44,4 \\
55,6\end{array}$ & $\begin{array}{l}39,6 \\
60,4\end{array}$ & $\begin{array}{l}44,4 \\
55,6\end{array}$ \\
\hline
\end{tabular}

Fonte: Fundação $\operatorname{IBGE}^{8}(1999)$.

A Tabela 5 focaliza mudanças observadas quanto à participação de lipídios na oferta alimentar. No NorteNordeste nota-se que a ascensão dos lipídios na dieta decorre do aumento da oferta de ácidos graxos saturados e dos monoinsaturados e poliinsaturados. No CentroSul, o declínio na proporção de calorias lipídicas oculta, de fato, aumento na oferta de ácidos graxos saturados e redução dos poliinsaturados. Esse movimento reflete, em essência, as tendências opostas vistas quanto ao consumo de carnes (em ascensão) e de óleos e gorduras vegetais (em declínio). Embora a participação dos ácidos graxos saturados na dieta cresça nas duas regiões, é no Centro-Sul que o limite máximo de ingestão-10\% do total calórico - fica próximo de ser alcançado $(9,2 \% \mathrm{em}$ 1996). Quanto à relação ácidos graxos insaturados e ácidos graxos saturados, a tendência é de estabilidade no Norte-Nordeste e de diminuição (portanto desfavorável) no Centro-Sul. Como resultado essencialmente do declínio no consumo de ovos, a disponibilidade do colesterol na dieta declina no Centro-Sul e se eleva apenas ligeiramente no Norte-Nordeste. Nas duas regiões, entretanto, os valores alcançados excedem o limite máximo de $100 \mathrm{mg} / 1.000 \mathrm{kcal}$.

\section{DISCUSSÃO}

Destaca-se que os instrumentos de avaliação utilizados no presente trabalho e as pesquisas de orçamento familiar não permitem aferir a quantidade absoluta de alimentos consumida pelas famílias, mas tão somente a participação relativa de cada alimento no total de alimentos disponíveis para consumo no domicílio. As pesquisas de orçamento familiar não consideram os desperdícios e os alimentos descartados, nem levam em conta os alimentos consumidos fora de casa. Assim, fornecem indicações da adequação da composição da dieta familiar, mas não da quantidade total de alimentos consumida pelas famílias. ${ }^{8}$

A adequação do consumo calórico total nas POF de 1988 e de 1996 poderia ser apreciada a partir da avaliação do peso e da altura das crianças e dos adultos residentes

Tabela 5 - Participação relativa de ácidos graxos e de colesterol na disponibilidade total de energia. Áreas metropolitanas do Brasil, 1988 e 1996.

\begin{tabular}{cccrrrr}
\hline & \multicolumn{2}{c}{ Norte-Nordeste } & \multicolumn{2}{c}{ Centro-Sul } & \multicolumn{2}{c}{ Brasil } \\
\cline { 2 - 7 } Lipídios & 1988 & 1996 & 1988 & 1996 & 1988 & 1996 \\
\hline Ácidos graxos (\%): & & & & & & \\
. Saturados & 7,6 & 8,0 & 8,7 & 9,2 & 8,5 & 8,9 \\
Mono-insaturados & 6,4 & 6,7 & 7,8 & 7,6 & 9,6 & 7,4 \\
- Poli-insaturados & 6,7 & 7,1 & 10,3 & 9,2 & 10,7 & 109,8 \\
Colesterol (Mg/1.000 Kcal) & 114,5 & 118,9 & 108,8 & 107,6 & 109,5 & 109,8 \\
\hline
\end{tabular}

Fonte: Fundação IBGE $^{8}$ (1999). 
nos domicílios estudados, mas dados antropométricos não são habitualmente coletados em pesquisas de orçamentos familiares. Análises de inquéritos antropométricos realizados no Brasil em períodos próximos às duas $\mathrm{POF}$ evidenciam, em todas as regiões do País, diminuição da desnutrição em crianças e em adultos e aumento da obesidade em adultos, o que indica expansão da taxa média de adequação do consumo calórico da população. ${ }^{17,18} \mathrm{~A}$ aparente contradição entre a redução (Centro-Sul) ou estabilização (Norte-Nordeste) da disponibilidade per capita de energia e a progressão da taxa média de adequação do consumo calórico poderia ser explicada por uma diminuição das necessidades calóricas da população (decorrente de mudanças no perfil ocupacional e de lazer dos indivíduos) ou mesmo por uma maior proporção de refeições feitas fora do domicílio. A primeira explicação, embora plausível, carece de suporte empírico adequado, uma vez que não se dispõem de informações confiáveis sobre a evolução do padrão de atividade física da população brasileira. A segunda explicação tem algum suporte empírico no Centro-Sul, mas não no Norte-Nordeste. Nas áreas metropolitanas do Centro-Sul, entre 1988 e 1996, a proporção de gastos com alimentação fora do domicílio cresceu de 24,4\% para 26,1\%; nas áreas metropolitanas do Norte-Nordeste, a mesma proporção declinou de $25,6 \%$ para $21,7 \%{ }^{8}$

Em trabalho anterior ${ }^{16}$ sobre a evolução do consumo alimentar nas áreas metropolitanas brasileiras entre as décadas de 60 e 80, foram constatadas duas tendências majoritárias: 1) a expansão no consumo relativo de produtos de origem animal (sobretudo leite e derivados, mas também carnes e ovos) em detrimento de cereais, leguminosas e raízes e tubérculos; e 2) a substituição intensa de gorduras animais (banha, toucinho e manteiga) por óleos vegetais e margarinas. O resultado dessas mudanças para a saúde da população foi de modo geral positivo, uma vez que, ao final, tornou-se possível melhorar simultaneamente o aporte protéico da dieta (e de outros nutrientes essenciais como minerais e vitaminas, os quais, em geral, se apresentam em maiores concentrações nos produtos de origem animal) e a composição lipídica da dieta (aumento na relação entre ácidos graxos poliinsaturados e saturados e redução no conteúdo de colesterol). $\mathrm{O}$ aspecto negativo das mudanças foi o aumento exagerado no conteúdo lipídico total da dieta observado na Região Sudeste, onde, em 1988, a proporção de calorias lipídicas atingiu cerca de $30 \%$ ou o limite máximo do intervalo recomendado para esse indicador.

Entre 1988 e 1996, período recente focalizado no presente trabalho, observou-se intensificação do consumo relativo de carnes em todas as áreas metropolitanas do País. O consumo relativo de leite e de seus derivados prosseguiu aumentando, mas em velocidade reduzida com relação aos períodos anteriores, enquanto o consu- mo de ovos passou a declinar, sobretudo no Centro-Sul do País. Leguminosas, raízes e tubérculos prosseguiram sua trajetória descendente em todo País, enquanto cereais e derivados tenderam a se estabilizar no Centro-Sul ou mesmo a se elevar ligeiramente no Norte-Nordeste. Nos dois inquéritos manteve-se reduzida a participação de gorduras animais (banha, toucinho e manteiga) na dieta (menos de $1 \%$ do total calórico em todo o País). A participação de óleos e de gorduras vegetais mantevese constante no Norte-Nordeste (10\% do total calórico) e declinou intensamente no Centro-Sul (de 15,2\% para $12,9 \%$ do total calórico). Como resultado do novo padrão de evolução, prosseguiu em todo o País a tendência de melhoria observada anteriormente quanto ao aporte de proteínas e de outros nutrientes essenciais presentes nos alimentos de origem animal. Por outro lado, o esgotamento do processo de substituição de gorduras animais por óleos e gorduras vegetais, ao lado do aumento no consumo de carnes, contribuiu para reverter a melhoria anterior observada em relação à relação entre ácidos graxos poliinsaturados e saturados. O mesmo não se deu com relação ao consumo de colesterol apenas em face da redução intensa observada no consumo de ovos. Finalmente, a contribuição dos lipídios para a dieta reduziu-se ligeiramente no Centro-Sul, mantendo-se, entretanto, ainda muito próxima do limite máximo de 30\%, e aumentou ligeiramente (de 23\% para 23,8\%) nas áreas metropolitanas do Norte-Nordeste.

A tendência ascendente da proporção de calorias lipídicas na dieta do Norte e Nordeste, a manutenção desse indicador em torno de valores muito elevados no Centro-Sul, a persistência de valores elevados para o colesterol dietético, o aumento dos ácidos graxos saturados e a redução dos carboidratos complexos em todas as áreas metropolitanas do País, ao lado da estagnação ou da redução do consumo de leguminosas, verduras, legumes, frutas e sucos naturais e da ascensão do consumo já excessivo de açúcar refinado e refrigerantes, são, em linhas gerais, os traços marcantes e negativos da evolução do padrão alimentar observada entre as POF de 1988 e 1996. Padrão semelhante de evolução vem sendo descrito em diversos países desenvolvidos e anunciado como bastante provável para países em desenvolvimento. ${ }^{3}$

O declínio notável no consumo de ovos e a retração substancial no consumo de óleos e gorduras vegetais e, em menor escala, o recuo discreto na proporção total de calorias lipídicas, tendências observadas nas áreas metropolitanas do Centro-Sul do País, constituem os dados mais importantes revelados pelos inquéritos de 1988 e 1996. Razões relacionadas a preços relativos dos alimentos dificilmente justificariam a diminuição substancial no consumo de ovos e de óleos e gorduras vegetais. Entre os inquéritos, o preço dos ovos pouco variou e o preço 
do óleo de soja (principal componente do grupo de óleos e gorduras vegetais) declinou intensamente. ${ }^{8}$ Uma hipótese seria atribuir o declínio no consumo desses alimentos a uma atitude consciente da população das áreas mais desenvolvidas do País em prol de uma dieta mais saudável. A restrição a frituras e a redução no consumo de alimentos ricos em colesterol, que têm nos ovos o seu mais conhecido paradigma, são duas das mais frequientes mensagens educativas que vêm sendo divulgadas por profissionais de saúde e por meios de comunicação de massa. O último inquérito antropométrico realizado no País evidenciou na Região Sudeste, mas não na Região Nordeste, declínio inédito da obesidade na população feminina adulta pertencente

\section{REFERÊNCIAS}

1. Bowes AP, Church HN. Food values of portions commly used. 7th ed. Philadelphia, USA: LippincottRaven Publishers; 1998.

2. Bray GA, Popkin BM. Dietary fat intake does affect obesity! Am J Clin Nutr 1998;68:1157-73.

3. Drewnowski A, Popkin BM. The nutrition transition: new trends in the global diet. Nutr Rev 1997;55:31-43.

4. Fundação IBGE. Consumo alimentar; antropometria. Rio de Janeiro; 1977. [Estudo Nacional da Despesa Familiar - ENDEF, v. 1: dados preliminares, Regiões I, II, III, IV e V, t. 1].

5. Fundação IBGE. Pesquisa do orçamentos familiares POF 1987/88. Rio de Janeiro; 1991. vol 1.

6. Fundação IBGE. Tabela de composição de alimentos. $4^{\mathrm{a}}$ ed. Rio de Janeiro; 1996. [Estudo Nacional da Despesa Familiar - ENDEF].

7. Fundação IBGE. Pesquisa do orçamentos familiares 1995-1996: primeiros resultados. Rio de Janeiro; 1997.

8. Fundação IBGE. Pesquisa do orçamentos familiares 1987 e 1996 [on line]. Disponível em URL <http:// www.ibge.gov.br/sidra>. [1999 nov 30].

9. Fundação Getulio Vargas. Food consumption in Brazil: family budgest survey in the early 1960's. Jerusalem: Keter Press; 1970.

10. Helsing E, Becker W. Food and health data: their use in nutrition policy making. Copenhagen: World Health Organization; 1991. [WHO-Regional Publications, European Series; 34].

11. Intersalt Cooperative Research Group. Intersalt: an international study of electrolyte excretion and blood pressure. Results for 24 hour urinary sodium and potassium excretion. Brit Med J 1988;297:319-28.

12. James WPT, Ferro-Luzzi A, Isaksson B, Szostak WB, Gualda-Vargas P. Healthy nutrition: preventing nutrition-related diseases in Europe. Copenhagen: World Health Organization; 1988. [WHO-Regional Publications, European Series; 24]. aos estratos sociais de maior renda. ${ }^{18}$ Espera-se que a análise da evolução dos padrões alimentares da população metropolitana do País, segundo estratos de renda, a ser efetuada a partir das POF de 1988 e 1996, propicie elementos adicionais para uma melhor compreensão das modificações que foram apontadas no presente trabalho.

\section{AGRADECIMENTOS}

Ao analista de sistemas Fernão Dias de Lima e à assistente de pesquisa Silvia Cristina Konno da Faculdade de Saúde Pública da Universidade de São Paulo, pelo apoio prestado na programação e no processamento de dados.

13. Keys A, editor. Coronary heart disease in seven countries. Circulation 1970;41(4 Suppl 1):I1-I211.

14. Keys A, Menotti A, Aravanis C, Blackburn $\mathrm{H}$, Djordeirc BS, Buzina R et al. The seven countries study: 2289 deaths in 15 years. Prev Med 1984;13:141-54.

15. Mahan LK, Escott SS. Alimentos, nutrição e dietoterapia. 9a ed. São Paulo: Roca; 1998.

16. Mondini L, Monteiro CA. Mudanças no padrão de alimentação na população urbana brasileira (19621988). Rev Saúde Pública 1994;28:433-9.

17. Monteiro CA, Benicio MHD'A, Freitas ICM. Melhoria em indicadores da saúde associados à pobreza no Brasil dos anos 90: descrição, causas e impacto sobre desigualdades regionais. São Paulo: NUPENS/USP; 1997.

18. Monteiro CA, Conde WL. A tendência secular da obesidade segundo estratos sociais: Nordeste e Sudeste do Brasil, 1975-1989-1997. Arq Bras Endocrinol Metabol 1999; 43:186-94.

19. Souci SW, Fachmann W, Kraut H. Food composition and nutrition tables. 5th ed. Stuttgart, Germany: Medpharm Scientific Publishers; 1994.

20. Stamler J, Shekelle R. Dietary cholesterol and human coronary heart disease: the epidemiological evidence. Arch Pathol Lab Med 1988;112:1032-40.

21. [WHO]. World Health Organization. Study Group on Diet, Nutrition and Prevention of Chronic Diseases; 1989; Geneva. Report. Geneva: World Health Organization; 1990. [WHO - Technical Report Series, 797].

22. [WHO]. World Health Organization. O besity: preventing and mananging the global epidemic: report of a WHO Consultation on O besity. Geneva: World Health Organization; 1998. (WHO/NUT/NCD 98.1)

23. World Cancer Research Fund. Food, nutrition and the prevention of cancer: a global perspective. Washington (DC): World Cancer Research Fund; 1997. 